\title{
The human prolactin gene upstream promoter is regulated in lymphoid cells by activators of T-cells and by cAMP
}

\section{G H Reem ${ }^{1}$, D W Ray ${ }^{2}$ and J R E Davis ${ }^{2}$}

\author{
${ }^{1}$ New York University Medical Center and The Kaplan Comprehensive Cancer Center, \\ 550 First Avenue, New York, New York 10016, USA \\ ${ }^{2}$ Endocrine Sciences Research Group, Department of Medicine, University of Manchester, \\ Manchester M13 9PT, UK
}

(Requests for offprints should be addressed to G H Reem)

\begin{abstract}
Prolactin (PRL) is produced in human thymocytes, $\mathrm{T}$-cells and endometrium. In these extrapituitary tissues, PRL gene transcription is directed by an alternative upstream promoter, and it is thought to act as a locally produced cytokine, with relevance for immune regulation and modulation of $\mathrm{T}$-cell function. We have studied PRL transcriptional regulation in the human T-lymphoblastoid Jurkat cell line transfected with a fragment of the upstream promoter linked to luciferase. A cAMP analogue (cptAMP) increased promoter activity rapidly and dose dependently. This increase was resistant to inhibition by cyclosporin $\mathrm{A}$ and thus independent of calcineurin phosphatase $(\mathrm{CN})$. T-cell activation by phorbol myristate acetate (PMA) failed to enhance promoter activity but phytohaemagglutinin (PHA) alone or PHA+PMA increased it, and cptAMP acted in synergy with PMA or PHA to increase it further. H-89, a cAMP-dependent protein kinase A
\end{abstract}

(PKA) inhibitor, inhibited the effect of cptAMP as did transfection with protein kinase inhibitor PKI, an expression vector of the specific inhibitor of PKA. A single point mutation in the CRE (cAMP response element) located at $-25 \mathrm{bp}$ in the $\mathrm{PRL}$ upstream promoter (TGACGT to TGCCGT) failed to reduce the response to cptAMP, while mutations or deletion of four nucleotides in the CRE to TACTCT diminished the response to cAMP by more than half.

We conclude that activity of the human PRL upstream extrapituitary promoter can be induced by activators of T-cells, as well as by a cAMP analogue. The signal is transmitted by PKA and the effect of cAMP is independent of CN. It is partly dependent on an intact proximal CRE motif but a more upstream enhancer may contribute to promoter regulation.

Fournal of Molecular Endocrinology (1999) 22, 285-293

\section{INTRODUCTION}

Prolactin (PRL) is produced in the pituitary and in extrapituitary tissues (Ben-Jonathan et al. 1996). The hormone synthesized in tissues other than the pituitary is identical in structure and function to that of the pituitary, but it is transcribed from an alternative upstream promoter. The human PRL (hPRL) gene promoter utilized by extrapituitary tissue is of interest in view of the autocrine and paracrine functions of PRL in immune cells, the endometrium, the mammary gland and the prostate (Henninghausen et al. 1997, Liu et al. 1997). PRL has a significant role in thymic development and in the regulation of the immune response, and may have a role in the pathogenesis or progression of autoimmune diseases (reviewed in Ben-Jonathan et al. 1996). The PRL receptor belongs to the cytokine, haematopoietic, growth hormone receptor super-family and is expressed on lymphoid cells, endometrium, prostate and other tissues (Ben-Jonathan et al.1996, Ferrag et al. 1996, Goffin et al. 1996, Socolovsky et al. 1997). PRL can therefore be regarded as a cytokine with pleiotropic function. The upstream promoter utilized in human lymphoid cells of T-cell origin (here referred to as the tPRL promoter) has as yet not been extensively studied.

The extrapituitary transcript of the hPRL gene is 150 nucleotides longer than that of the pituitary PRL mRNA due to a $5^{\prime}$ non-coding exon (exon 1a) (Hiraoka et al. 1991). A portion of this untranslated sequence is unique to the extrapituitary mRNA. The start site of exon $1 \mathrm{a}$ is located $5.8 \mathrm{~kb}$ upstream 
of the pituitary start site and the extrapituitary transcription of the gene is directed by an upstream promoter (DiMattia et al. 1990, Berwaer et al. 1994, Gellersen et al. 1994). PRL expression in nonpituitary tissues differs from that of the pituitary in that it is cell type specific and independent of the transcription factor Pit-1 (Berwaer et al. 1994, Gellersen et al. 1994). After transient transfection in lymphoid cells, the upstream promoter directs reporter gene expression in both PRL-secreting and non-secreting cells, whereas in endometrial cells only differentiated stromal cells which secrete PRL activate the upstream promoter (Berwaer et al. 1994, Gellersen et al. 1994). On the other hand, the upstream promoter is not activated when transfected into pituitary lactotrophs or HeLa cells, indicating that the mechanisms regulating PRL gene expression are cell type specific with a selection between alternate promoters in pituitary or extrapituitary sites of PRL production (Berwaer et al. 1994).

The present study was designed to investigate the regulation of the hPRL upstream promoter in a T-lymphoid Jurkat cell line. We have found that these cells readily express a reporter gene linked to a promoter fragment comprising the proximal $375 \mathrm{bp}$ upstream from the start site of the upstream exon 1a promoter (tPRL 375-luc). Activation of protein kinase $\mathrm{C}$ (PKC) by phorbol myristate acetate (PMA) without costimulation with phytohaemagglutinin (PHA) was insufficient to induce reporter gene expression, while PHA by itself was only weakly stimulatory. By contrast we found that the longacting analogue of cAMP, chlorophenylthio-cAMP (cptAMP), was sufficient to activate the upstream promoter within $2 \mathrm{~h}$. Activation by cptAMP synergized with that of PMA and/or PHA. Investigation of the transduction of the signal elicited by cptAMP showed that it was mediated by protein kinase A (PKA), but not by calcineurin phosphatase $(\mathrm{CN})$. Mutation or deletion of $4 \mathrm{bp}$ in the near consensus cAMP response element (CRE) partly diminished the activation of the upstream PRL promoter suggesting the participation of another more distal enhancer in addition to the CRE located close to the TATA box. These findings reveal possible mechanisms for the regulation of lymphocyte PRL production, which may be relevant for paracrine modulation of immune responses.

\section{METHODS}

\section{Cell culture, transient transfections and luciferase assay}

Jurkat cells growing in log phase in complete RPMI 1640 medium containing $10 \%$ fetal calf serum
(Gibco Laboratories, Grand Island, NY, USA) were washed with serum-free RPMI 1640, resuspended in medium $\left(5 \times 10^{7} / \mathrm{ml}\right)$ and transiently transfected by electroporation, $320 \mathrm{~V}, \quad 960 \mu \mathrm{F}$ capacitance (BioRad Pulser Apparatus, BioRad, Richmond, CA, USA). Cells were resuspended in $15 \mathrm{ml}$ complete medium and incubated overnight or as indicated. Following incubation, cells were divided into $1.0 \mathrm{ml}$ aliquots $\left(5 \times 10^{4}\right.$ cells $\left./ \mathrm{ml}\right)$ and induced with the appropriate reagents. After induction for varying periods of time (as indicated), cells were harvested, lysed in lysis buffer (Promega kit, Promega, Madison, WI, USA) and lysates were frozen at $-80{ }^{\circ} \mathrm{C}$. Lysates were assayed for luciferase activity in triplicate with the Promega reagent kit, following the instructions of the manufacturer. Light intensity was read in a luminometer (Lumat LB 9501, Berthold, Bad Wildban, Germany). Proteins were determined with the BioRad reagents. Data were calculated as luciferase activity per mg protein and results expressed as fold induction relative to the uninduced samples.

\section{Reagents}

cptAMP was purchased from Boehringer Mannheim (Indianapolis, IN, USA) forskolin, isobutyl-1-methylxanthine (IBMX), H-89 (N-(2- $\beta$ bromocinnamylaminoethyl)-5-isoquinolinesulphonamide) and PMA were from Sigma Chemical Co. (St Louis, MO, USA). PHA was from ICN (Costa Mesa, CA, USA) and cyclosporin A (CsA) was a gift from Sandoz Pharmaceuticals, Basel, Switzerland.

CREB-1 bZIP represents the $14 \mathrm{kDa}$ DNAbinding and dimerization domains of CREB (CRE binding protein) corresponding to amino acids 254-327. The protein was produced in Escherichia coli and purified by column chromatography (Santa Cruz Biotechnology, Santa Cruz, CA, USA).

\section{Plasmids}

tPRL 375-luc construction has been described in detail (Berwaer et al. 1994). The oligonucleotide primers used for the mutations were synthesized with a 3984 DNA synthesizer (Applied Biosystems, Foster City, CA, USA). They contained the following mutations: TGACGTT, located at $-25 \mathrm{bp}$ upstream from the start site of exon $1 \mathrm{a}$, to TGCCGTT, a single point mutation; mutations of four nucleotides in the CRE to TACTCTT; and deletions of these four nucleotides. The nucleotide primers, each complementary to opposite strands of the vector, were $43 \mathrm{bp}$ in length and the mutations were situated in the middle of the primer. 

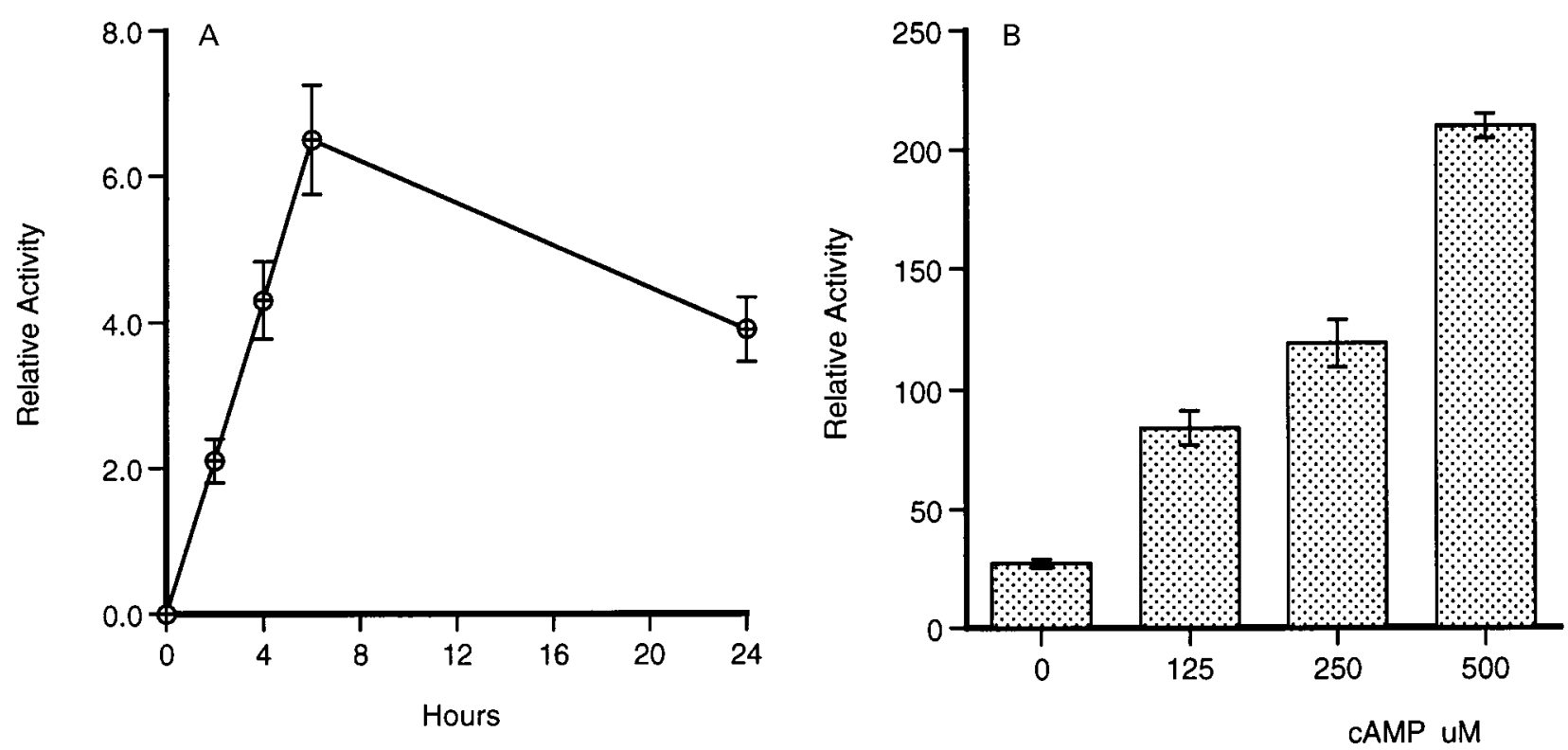

FIGURE 1. Kinetics of activation of tPRL transcription by cptAMP. (A) Jurkat cells were transiently transfected with tPRL 375-luc and $250 \mu \mathrm{M}$ cptAMP was added $16 \mathrm{~h}$ later. Cells were harvested 2, 4, 6 and $24 \mathrm{~h}$ after addition of cptAMP. Induction was apparent after $2 \mathrm{~h}$, peaked at $6 \mathrm{~h}$ and subsequently decreased. At $24 \mathrm{~h}$, promoter activation was decreased. (B) PRL promoter activation increased with increasing concentrations of cptAMP. Reporter gene activation by cptAMP is expressed as fold induction relative to unstimulated control values. Data shown are means \pm S.D. of three independent experiments.

Mutations in tPRL 375-luc were constructed with the QuikChange site-directed mutagenesis kit (Stratagene, La Jolla, CA, USA), according to the manufacturer's instructions. All constructs were verified by sequencing with the 373 DNA sequencer. Protein kinase inhibitor PKI and its inactive mutant PKIm were a gift from Dr R A Maurer (Oregon Health Sciences, Portland, OR, USA) (Day et al. 1989).

\section{Gel retardation assay}

Double-stranded oligonucleotides were synthesized representing the putative CRE located around $-25 \mathrm{bp}$ in the hPRL upstream promoter, or containing a single point mutation, or with a $4 \mathrm{bp}$ deletion, as used in the transfection studies. A consensus CRE was used for comparison. The sequences are given below (sense strand only, CRE element in bold):

hPRL-CRE: 5'-C'TG AAG CAG GCC ATA AAA GAA TCC TCT GAC GTT TCT ATA AAG TAG G-3'

hPRLmut-CRE (single base mutation): 5'-CTG AAG CAG GCC ATA AAA GAA TCC TCT GCC GTT TCT ATA AAG TAG G-3'

hPRLdel-CRE: 5'-CTG AAG CAG GCC ATA AAA GAA TCC TCT----TT TCT ATA AAG TAG G-3'
Consensus CRE: 5'-AGA GAT TGC CTG ACG TCA GAG AGC TAG-3'

Double stranded oligonucleotides were created by annealing at $95^{\circ} \mathrm{C}$ for $2 \mathrm{~min}$ and cooling slowly. DNA was end-labelled using polynucleotide kinase (Promega) and labelled probes were purified by passing over Sephadex G50 columns in Tris-EDTA buffer. One hundred nanograms recombinant CREB-1 bZIP were incubated with $1 \mu \mathrm{g}$ poly-dI.dC in buffer ( $5 \mathrm{mM}$ Tris (pH7.5), $50 \mathrm{mM} \mathrm{NaCl}, 1 \mathrm{mM}$ dithiothreitol, $1 \mathrm{mM}$ EDTA, 5\% glycerol) with or without competitor DNA for $20 \mathrm{~min}$ at $37^{\circ} \mathrm{C}$. The binding reaction was initiated by adding 20000 c.p.m. labelled DNA (approximately $50 \mathrm{fmol}$ ). Incubation was continued for $15 \mathrm{~min}$ at $37^{\circ} \mathrm{C}$, and complexes were resolved on a $6 \%$ native acrylamide gel run in $0.5 \times$ TBE buffer. Gels were fixed, dried and exposed to film overnight at $-70{ }^{\circ} \mathrm{C}$.

\section{RESULTS}

Induction by cptAMP of PRL gene expression in transiently transfected Jurkat cells: kinetics of induction and dose response to cAMP

The response to cptAMP was studied in Jurkat cells transfected with the tPRL 375-luc reporter gene. 


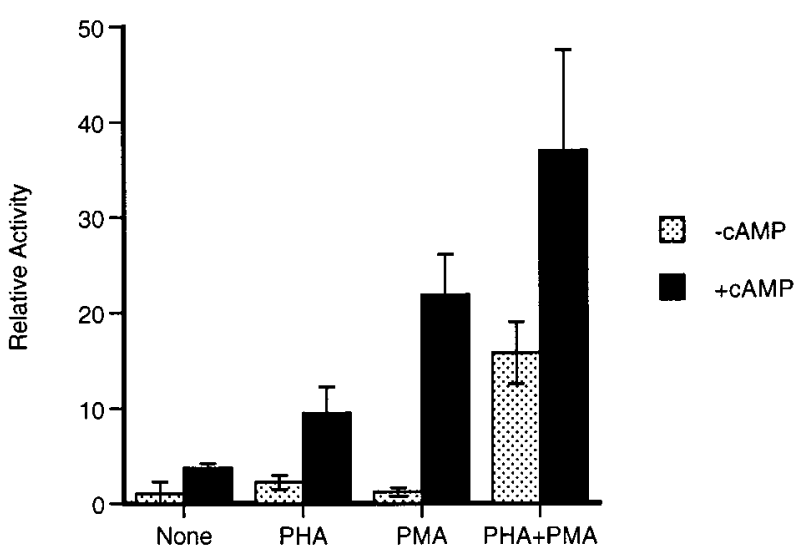

FIGURE 2. Effect of activators of T-cells and of cptAMP. Jurkat cells were transiently transfected with tPRL 375-luc and incubated with PHA, PMA, or PHA+PMA alone or in combination with or without cptAMP. Note that cptAMP acts in synergy with these activators. Data are presented as means \pm S.D. of three independent experiments.

Transcription of the reporter gene was rapid; it was apparent after $2 \mathrm{~h}$ and rose during the following $6 \mathrm{~h}$ (Fig. 1A). At $24 \mathrm{~h}$, transcription was still elevated, but lower than at $6 \mathrm{~h}$. Similar kinetics of response were observed by raising the intracellular cAMP concentration with forskolin and IBMX (data not shown). The response to cptAMP was dose dependent (Fig. 1B), and cptAMP had no effect on the control plasmid devoid of the hPRL promoter fragment (pXP-luc) (data not shown).

\section{Effect of activation of Jurkat cells on hPRL promoter expression in the presence and absence of cptAMP: synergy with activators of T-cells}

Since the activation of T-cells and thymocytes can induce the secretion of PRL (O'Neal et al. 1992, and unpublished data), we investigated the effect of PMA and PHA singly, in combination with each other, and in combination with cptAMP. PHA and PMA alone had little or no effect but the combination of PMA+PHA was synergistic, while cptAMP had further synergistic effects with both PMA and PHA, resulting in large inductions in promoter activity (Fig. 2).

\section{Effect of the inhibition of $\mathrm{CN}$ phosphatase by CsA on PRL promoter expression}

Since some effects of cptAMP are in part dependent on $\mathrm{CN}$, we studied the effect of the $\mathrm{CN}$ inhibitor $(50 \mathrm{ng} / \mathrm{ml})$ on cptAMP-induced hPRL promoter

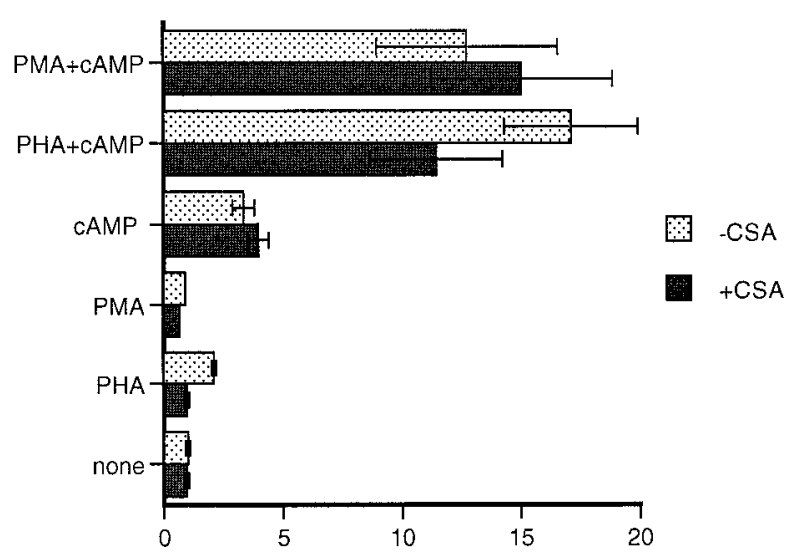

FIGURE 3. Effect of CsA $(50 \mathrm{ng} / \mathrm{ml})$ on the activation of the PRL promoter by cptAMP, PHA and

PHA+cptAMP. CsA alone had no effect on promoter activity, nor did it affect the activation by cptAMP alone or by PMA+cptAMP, whereas activation by PHA or by $\mathrm{PHA}+\mathrm{cptAMP}$ was inhibited by CsA. Data represent means \pm S.D. of three independent experiments. The horizontal axis shows fold increase in luciferase activity.

activity. We found that the signal for activation of the promoter by cptAMP and by PMA+cptAMP was resistant to inhibition by CsA and thus independent of $\mathrm{CN}$ (Fig. 3). CsA in this concentration is effective in suppressing PHA-induced transcription of the interleukin-2 (IL-2) promoter. When the CsA concentration was increased 10-fold $(500 \mathrm{ng} / \mathrm{ml})$ it still failed to reduce the transcription of the hPRL promoter (data not shown).

To further test the effect of CsA, experiments were carried out with cells activated by PHA and PHA+cptAMP. PHA is known to activate T-cells, to induce calcium flux, and thus sensitize $\mathrm{CN}$ to inhibition by CsA. In the presence of PHA, CsA inhibited the signalling pathway to the hPRL promoter (Fig. 3).

\section{Inhibitors of cAMP-dependent PKA: H-89 and PKI}

To determine whether the effect of cptAMP was dependent on cAMP-dependent PKA, we studied the effect of two inhibitors, the isoquinolinesulphonamide drug H-89 and PKI (Day et al. 1989). Cotransfection with a PKI expression vector $(4 \mathrm{ng} / \mathrm{ml})$ inhibited the response to cptAMP; increasing the amount of cotransfected PKI to $8 \mathrm{ng} / \mathrm{ml}$ had no further effect. The inactive mutant PKI expression vector had no effect in control experiments (Fig. 4A). H-89 had no effect on basal promoter activity, but diminished the response to cptAMP without affecting cell viability (Fig. 4B). 

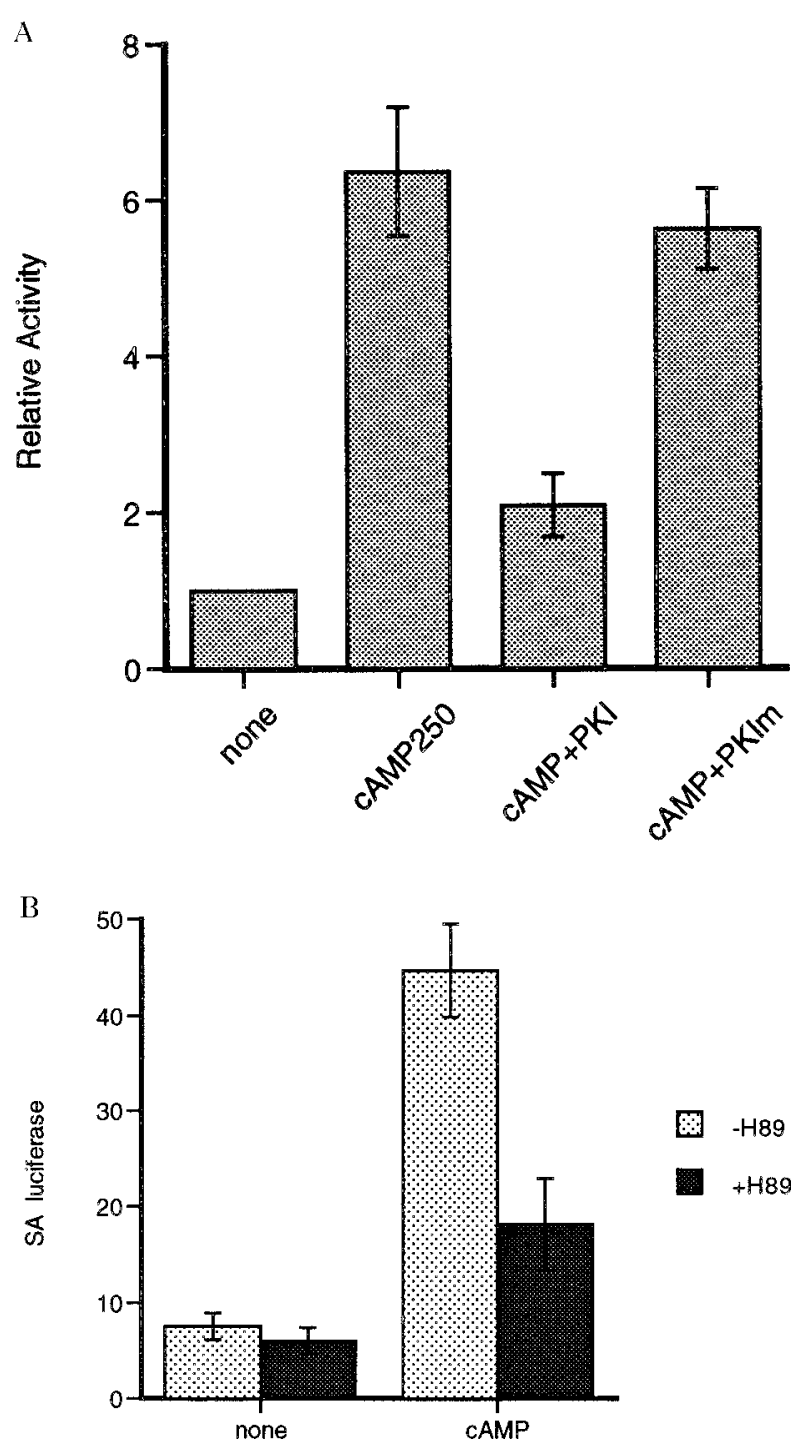

FIGURE 4. Effect of inhibition of PKA. (A) Jurkat cells were transiently cotransfected with tPRL 375-luc and the PKA-inhibitor expression vector PKI, or its inactive mutant PKIm, and treated with cptAMP $(250 \mu \mathrm{M})$ as indicated. Note that PKI was inhibitory, whereas PKIm was not. (B) Jurkat cells were transfected with tPRL 375 -luc and incubated for $24 \mathrm{~h}$, and treated with $2 \mu \mathrm{M}$ H-89 for $6 \mathrm{~h}$ before cell harvest. Cell viability was unaffected by H-89 (69\% compared with $72 \%$ in control) or by cptcAMP or cptAMP+H-89 (78 and 75\%; respectively). Data represent means \pm S.D. of three independent experiments.

\section{Role of the CRE in mediating activation of the PRL promoter by cptAMP}

Having established the role of PKA in transmitting the signal generated by cptAMP, we examined the effect of mutations and deletions in a proximally located CRE in the tPRL promoter. The upstream promoter does not contain the classical palindrome TGAC GTCA, but possesses the incomplete sequence TGAC GTTT, which is located $25 \mathrm{bp}$ upstream of the start site of exon 1a. We generated the following mutations in tPRL 375-luc and tested their effect on the induction of the promoter in comparison with that of the wild type: (i) point mutation: TGAC GTT to TGCC GTT; (ii) $4 \mathrm{bp}$ substitution: TGAC GTT to TACT CTT; (iii) 4 bp deletion: TGAC GTT to T-TT

The point mutation in the CRE had no effect on the activation of the promoter by cptAMP (data not shown). By contrast, substitution or deletion of $4 \mathrm{bp}$ inhibited the response to cptAMP, to cptAMP+PHA and to cptAMP+PMA by more than half. Synergy of cptAMP with PHA and with PMA was preserved, in the mutants, indicating that the response to cAMP and the synergistic response of cAMP with T-cell activators was not entirely dependent on an intact CRE motif (Fig. 5).

The results shown in Figs 4 and 5 provide evidence that the effect of cptAMP is mediated by PKA and is in part dependent on the CRE since mutation or deletion of the CRE diminishes the activation of the promoter by more than $50 \%$.

\section{Binding of CREB to the hPRL CRE}

The binding of CREB to the putative CRE in the hPRL gene (hPRL-CRE) was studied by a gel retardation assay. The wild type hPRL sequence was able to bind recombinant CREB fragment, and the binding was specifically competed both by a homologous sequence and by a consensus CRE oligonucleotide (Fig. 6, lanes 3 and 4). Under these conditions no competition for protein binding was exerted by 100 -fold excess of oligonucleotides containing either a single base mutation in the hPRL-CRE or a $4 \mathrm{bp}$ deletion (lanes 5 and 6). However, when the mutated hPRL-CRE oligonucleotides were used as labelled target DNA, the 4 bp deletant was unable to bind CREB fragment at all (lane 9), whereas the single point mutant hPRL-CRE oligonucleotide exhibited definite, though weak, binding (lane 8). These results therefore confirm that the hPRL sequence and the single point mutation are able to bind CREB, and that the single point mutation binds with lower efficiency than the wild type.

\section{DISCUSSION}

In this study the regulation of the upstream hPRL promoter utilized in lymphoid cells of T-cell origin 


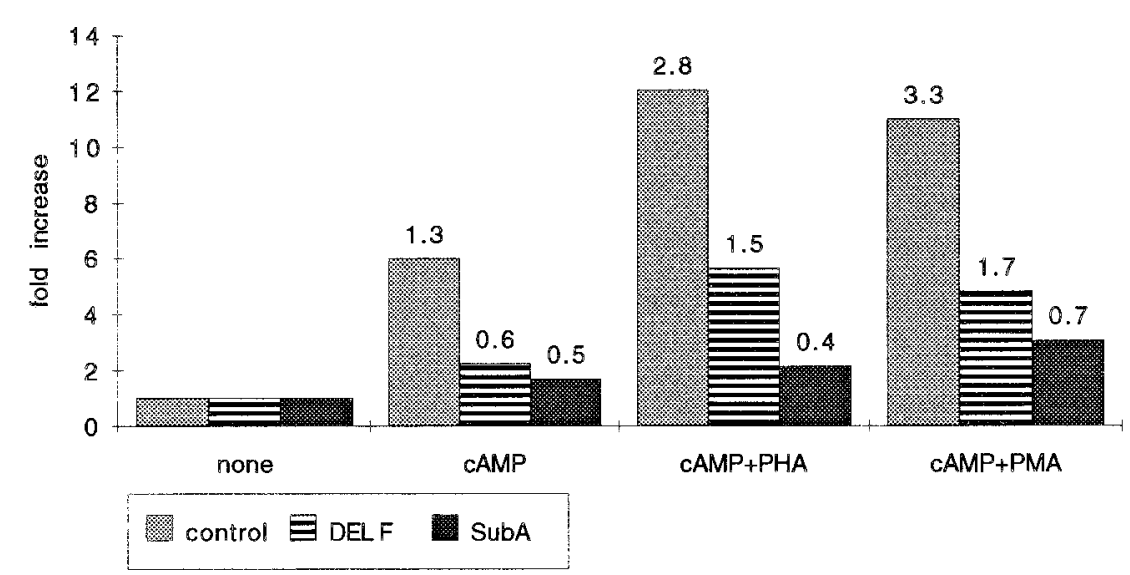

FIGURE 5. Effect of mutation in the tPRL CRE. Jurkat cells were cotransfected with the wild type tPRL 375-luc reporter gene, and with the mutant constructs described in Materials and Methods, and treated with cptAMP, cptAMP+PHA and cptAMP+PMA for $6 \mathrm{~h}$ before harvest. Induction of promoter activity was reduced in the mutants with deletions and in mutants with substitutions in CRE. Data represent the means \pm s.D. of three experiments. The numbers above the bars represent the s.D. The basal expression of the mutants was no lower than that of the control. Transfection efficiency was similar as determined by cotransfection with $\beta$-galactosidase in these experiments.

(tPRL promoter) was investigated. The tPRL promoter is activated in some extrapituitary tissues; it is located in exon $1 \mathrm{a}$, which starts $5.8 \mathrm{~kb}$ pairs upstream of the pituitary start site (Hiraoka et al. 1991). In the present study Jurkat T-cells were transiently transfected with a promoter fragment comprising the proximal $375 \mathrm{bp}$ from the upstream promoter linked to a luciferase reporter gene, tPRL 375-luc.

We found that the kinetics of activation of the upstream PRL promoter of lymphoid cells differ from those observed in endometrial stromal cells (Gellersen et al. 1994, Telgmann et al. 1997). This is evidenced by our observation that, in cultures of Jurkat cells, tPRL promoter activation by a stable analogue of cAMP occurs rapidly, within $2 \mathrm{~h}$, and peaks at $6 \mathrm{~h}$, then decreases but is still observed at $24 \mathrm{~h}$ (Fig. 1A). This response to cptAMP is dose dependent (Fig 1B), and has also been observed in cultures activated with forskolin, an activator of adenyl cyclase, and IBMX, an antagonist of cAMP phosphodiesterase (data not shown). By contrast, in undifferentiated endometrial stromal cells the upstream promoter is not immediately activated by cptAMP. Only if differentiation is induced by pre-incubation with a long-acting analogue of cAMP is promoter activation observed (Gellersen et al. 1994, 1995, 1997, Telgmann et al. 1997). According to Telgmann et al., activation of the promoter in endometrial cells has two distinct phases, a weak, early one which is apparent at $6 \mathrm{~h}$ and a strong late phase lasting for $24 \mathrm{~h}$. During the late phase, activation continues to increase. This is in contrast to the rapid response in lymphoid cells, which lack a late phase activation. The major transcriptional response observed in continuously activated endometrial stromal cells is mediated by an enhancer postulated to be located between -270 and $-332 \mathrm{bp}$ (Telgmann et al. 1997). This enhancer apparently differs from the consensus response element CRE situated in proximity of the TATA box which mediates the immediate response observed in lymphoid cells. T-cell activation by the $\mathrm{T}$-cell receptor, or by a lectin and a second inducer, is associated with a rise in intracellular cAMP and intracellular calcium preceding IL-2 secretion and phosphorylation of a serine protein phosphatase (Trachman et al. 1995, Feuerstein et al. 1996). Activation of Jurkat cells by either PHA or PHA+PMA results in activation of the tPRL promoter. Activation by either PHA or PMA synergizes markedly with the effect of cptAMP in the native as well as in the mutated promoter sequences (Figs 2 and 5).

cAMP is a universal second messenger which can control the expression of target genes either positively or negatively, due to the binding of specific transcription factors to promoters of these genes. Activation of gene transcription mediated by PKA has been studied in detail (Brindle et al. 1995, 

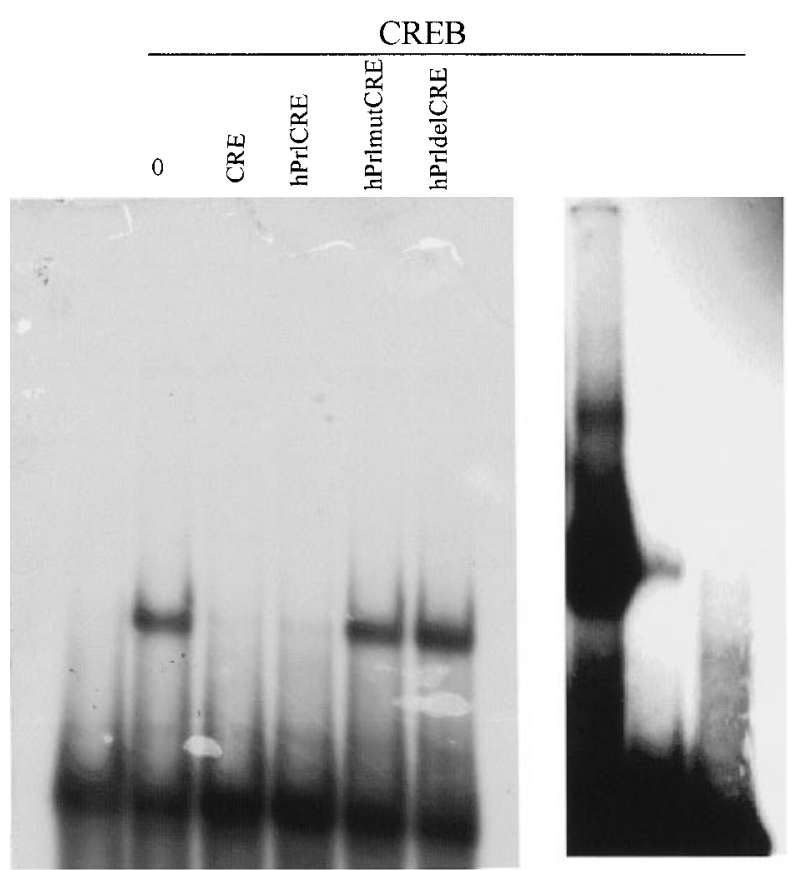

hPrl CRE

\section{protein}

competitor

\section{CREB}

\section{Free Probe}

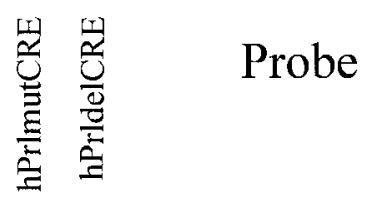

FIGURE 6. Binding of the putative hPRL-CRE and its mutants to recombinant CREB-1 bZIP by gel retardation assay. Lanes $1-6$, the radiolabelled hPRL-CRE (wild type) is used as target DNA. Lane 1, no added CREB protein; lanes 2-6, CREB protein (100 ng); lane 2, no competitor; lane 3, competition with $100 \times$ consensus CRE; lane 4 , competition with $100 \times$ hPRL-CRE (wild type); lane 5 , competition with $100 \times$ hPRLmut-CRE (single base mutant); lane 6 , competition with $100 \times$ hPRLdel-CRE (4 bp deletant). Lanes 7-9, wild type or mutant hPRL-CRE target sequences and CREB protein (1000 ng). Lane 7, hPRL-CRE (wild type); lane 8, hPRLmut-CRE (single base mutant); lane 9, hPRLdel-CRE (4 bp deletant).

Telgmann et al. 1997). Following binding of cAMP to the regulatory subunit of PKA, the catalytic subunit of PKA is released from the holoenzyme and translocates to the nucleus where it phosphorylates and activates CREB/ATF proteins. Phosphorylation of CREB facilitates the binding of transcription factors such as CREB binding protein to interact with the transcription complex. Phosphorylation of CREB is insufficient to activate transcription (Brindle et al. 1995). $\mathrm{Ca}^{2+}$ and PKC and other kinases can also phosphorylate CREB independently of PKA, but two interacting stimuli, one of them being PKA, are required to initiate transcription (Liu et al. 1993, Thompson et al. 1995). Transcription is further regulated by an interplay between kinases and phosphatases.
The expression and secretion of $\mathrm{IL}-2$ is inhibited by cAMP and this inhibitory signal is in part mediated by $\mathrm{CN}$, a calcium/calmodulindependent serine phosphatase (Muramatsu \& Kincaid 1996, Paliogianni et al. 1996) and by inducible cAMP early repressor, a cAMPinducible transcriptional repressor which binds to nuclear factor activating T-cells (NF-AT)/AP1 motifs. Binding to NF-AT can be competed by oligonucleotides containing CRE motifs (Bodor et al. 1996, Bodor \& Habener 1998). By contrast with its inhibitory action on the IL-2 promoter, we found that cptAMP activates the tPRL promoter. Activation of tPRL by cptAMP is independent of CN and IL-2, but can be increased by activators of T-cells. 
We tested the role of $\mathrm{CN}$ in transmitting the signal mediated by cAMP, by inhibiting $\mathrm{CN}$ with CsA and determining tPRL promoter activation. CsA, an immunosuppressive agent, forms a complex with its binding protein cyclophilin and inhibits the catalytic unit of CN (ElRouby et al. 1992, Reem 1992). The signal pathway elicited by cAMP differs according to cell type, the stage of development and the genetic make up of the cell, as well as according to the signal by which the target gene is induced. We observed that CsA in Jurkat cells, in a concentration sufficient to abrogate the activation of NF-AT (ElRouby et al. 1992, Reem 1992, Reem et al. 1993), did not inhibit the tPRL promoter activity induced with cptAMP or with the combination of PMA plus cptAMP. Resistance to $\mathrm{CsA}$ indicates that this signal pathway is independent of CN. Since cptAMP also inhibits the IL-2 promoter (Muramatsu \& Kincaid 1996, Paliogianni et al. 1996) it can be inferred that tPRL promoter activation by cptAMP is also independent of IL-2. IL-2 did not reverse the effect of cptAMP (data not shown). By contrast, CsA inhibited the response elicited by PHA+cptAMP, since activation by PHA is dependent on $\mathrm{Ca}^{2+}$ and $\mathrm{CN}$ (Fig 3). The role of PKA in mediating the signal for cptAMP in the activation of the PRL promoter was demonstrated by the finding that both PKI and H-89 inhibit the activation of the promoter by cptAMP (Fig. 4).

The tPRL promoter has a CRE motif at bp -25 . This motif TGACGTTT differs from the classic motif TGACGTCA, but is identical with the CaRE motif described in the c-fos promoter (Sheng et al. 1990, Liu et al. 1993). Substitution of TGAC GTT to TACT CTT or deletion of ACTC reduced promoter activation by cAMP alone, by cAMP+ PHA and by CAMP+PMA by more than half, but synergy of cAMP with PHA and PMA was preserved (Fig. 5). These observations indicate that activation of T-cells contributes to the response of the promoter and further suggests that transcription is in part dependent on this CRE motif and on an interplay with a more distal enhancer in the promoter region.

The role of the putative CRE in the hPRL gene (hPRL-CRE) was investigated by studying the binding of CREB in vitro by the gel retardation assay with wild type and mutated hPRL-CRE oligonucleotides as labelled target DNA. The DNA-protein binding data indicate that CREB is able to bind to the native hPRL CRE-like sequence. This binding was abolished by the $4 \mathrm{bp}$ deletion, and reduced but still demonstrable with the single base substitution. Hence it is likely that the cAMP induction of hPRL promoter activity seen in the transfection studies involves transcriptional acti- vation by either CREB or a CREB family member in conjunction with a more distal enhancer.

In conclusion, we have provided evidence that the hPRL gene upstream promoter in Jurkat lymphoblastoid cells is induced by the activators of T-cells PHA and PMA+PHA, which synergize with cAMP. Activation of the tPRL promoter in lymphoblastoid cells differs from that in endometrial cells by the kinetics of activation. Late phase activation, as observed in endometrial cells, is not seen in lymphoid cells. Activation of transcription of the tPRL promoter in T-cells by cAMP and $T$-cell activators is partly dependent on an intact CRE motif and on binding of CREB or a CREB family member.

Since regulation of PRL expression by activators of T-cells may influence the cellular immune response, this observation supports the notion that PRL has an immunomodulatory function. Aberrant regulation of PRL could contribute to other predisposing factors in the development of autoimmune disease.

\section{ACKNOWLEDGEMENTS}

This study was supported by National Institute of Health grant RO I CA 33653 awarded to G H R and by the Comprehensive Cancer Center Core Grant 16087 to New York University Medical Center. We thank Ms Min Zhao for technical assistance, Dr Jan Sap for useful discussions and for careful reading of the manuscript, Dr R A Maurer for his gift of the PKI and PKIm plasmids, and Dr Bernard Goldschmidt for preparation of the oligonucleotides and for sequencing the mutated plasmids. Our thanks to Ms J Small for the preparation of the manuscript.

\section{REFERENCES}

Ben-Jonathan N, Mershon JL, Allen DL \& Steinmetz RW 1996 Extrapituitary prolactin: distribution, regulation, functions, and clinical aspects. Endocrine Reviews 17 639-669.

Berwaer M, Martial JA \& Davis JRE 1994 Characterization of an up-stream promoter directing extrapituitary expression of the human prolactin gene. Molecular Endocrinology $\mathbf{8}$ 635-642.

Bodor J \& Habener JF 1998 Role of transcriptional repressor ICER in cyclic AMP-mediated attenuations of cytokine gene expression in human thymocytes. Fournal of Biological Chemistry 273 9544-9551.

Bodor J, Spetz A-L, Strominger JL \& Habener JF 1996 cAMP inducibility of transcriptional repressor ICER in developing and mature human T lymphocytes. Proceedings of the National Academy of Sciences of the USA 93 3536-3541. 
Brindle P, Nakajima T \& Montiminy M 1995 Multiple protein kinase A-regulated events are required for transcriptional induction by cAMP. Proceedings of the National Academy of Sciences of the USA 92 10521-10525.

Day RN, Walder JA \& Maurer RA 1989 A protein kinase inhibitor gene reduces both basal and multihormonestimulated prolactin gene transcription. Fournal of Biological Chemistry 264 431-436.

DiMattia GE, Gellersen B, Duckworth LM \& Friesen GH 1990 Human prolactin gene expression: use of an alternative noncoding exon in decidua and the IM-9-P3 lymphoblast cell line. Fournal of Biological Chemistry 256 16412-16421.

ElRouby S, Shi Y \& Reem GH 1992 Comparison of the properties of the CsA analogs monoacetyl CyC (O-acetylthreonine $^{2}$ cyclosporine) and methyl-alanyl CsA (N-methylL-alanyl $1^{6}$ cyclosporin). Monoacetyl cyclosporin is immunosuppressive without binding to cyclophilin. Clinical and Experimental Immunology 89 136-142.

Ferrag F, Chiarenza A, Goffin V \& Kelly PA 1996 Convergence of signalling transduced by prolactin (PRL)/ cytokine chimeric receptors on PRL-responsive gene transcription. Molecular Endocrinology 10 451-460.

Feuerstein N, Firestein R, Aiyar N, He X, Murasko D \& Cristofalo V 1996 Late induction of CREB/ATF binding and a concomitant increase in cAMP levels in T and B lymphocytes stimulated via the antigen receptor. Fournal of Immunology 156 4582-4593.

Gellersen B, Kempf T, Telgmann R \& DiMattia GE 1994 Nonpituitary human prolactin gene transcription is independent of Pit-1 and differentially controlled in lymphocytes and endometrial stroma. Molecular Endocrinology 8 356-373.

Gellersen B, Kempf R, Telgmann R \& DiMattia GE 1995 Pituitary-type transcription of the human prolactin gene in the absence of Pit-1. Molecular Endocrinology 9 887-901.

Gellersen B, Kempf R \& Telgmann R 1997 Human endometrial stromal cells express novel isoforms of the transcriptional modulator CREM and up-regulate ICER in the course of decidualization. Molecular Endocrinology 11 97-113.

Goffin V, Bole-Feysot C, Ferrag F, Maaskant R, Vincent V, Weimann E \& Kelly PA 1996 Signal transduction of prolactin and cytokine receptors. In Breast Cancer, Advances in Biology and Therapeutics, pp 131-137. Eds F Calvo, M Crepin \& H Magdelena. Montrouge, France: John Libbey Eurotext Ltd.

Henninghausen L, Robinson GW, Wagner K-U \& Liu X 1997 Prolactin signalling in mammary gland development. Fournal of Biological Chemistry 272 7567-7569.

Hiraoka Y, Shiozawa M, Aiso S, Fukasawa T, Yasuda M \& Miyai K 1991 A placenta-specific 5' non-coding exon of human prolactin. Molecular and Cellular Endocrinology 75 71-80.

Liu F, Thompson A, Wagner S \& Greenberg ME 1993 Activating transcription factor-1 can mediate $\mathrm{Ca}^{2+}-$ and cAMP-inducible transcriptional activation. Fournal of Biological Chemistry 268 6714-6720.

Liu X, Robinson GW, Wagner K-U, Garrett L, WynshawBoris A \& Hennighausen L 1997 Stat5a is mandatory for adult mammary gland development and lactogenesis. Genes and Development 11 179-186.

Muramatsu T \& Kincaid RL 1996 Inhibition of NF-AT signal transduction events by a dominant-negative form of calcineurin. Biochemical and Biophysical Research Communications 218 466-472.

O’Neal KD, Montgomery DW, Truong TM \& Yu-Lee L-Y 1992 Prolactin gene expression in human thymocytes. Molecular and Cellular Endocrinology 87 R19-R23.

Paliogianni F, Hama N, Mavrothalassitis GJ, Thyphronitis G \& Boumpas DT 1996 Signal requirements for interleukin 4 promoter activation in human T cells. Cellular Immunology $16833-38$.

Reem GH 1992 Molecular mode of action of cyclosporine and FK 506 in human thymocytes. Fournal of Autoimmunity $\mathbf{5}$ (Suppl A) 159-165.

Reem GH, ElRouby S \& Shi Y 1993 Study of the immunosuppressive properties of cyclosporine analogs. Annals of the New York Academy of Sciences 685 336-338.

Sheng M, McFadden G \& Greenberg ME 1990 Membrane depolarization and calcium induce $c$-fos transcription via phosphorylation of transcription factor CREB. Neuron 4 571-582.

Socolovsky M, Dusanter-Fourt I \& Lodish HF 1997 The prolactin receptor and severely truncated erythropoietin receptors support differentiation of erythroid progenitors. Fournal of Biological Chemistry 272 14009-14012.

Telgmann R, Maronde E, Tasken K \& Gellersen B 1997 Activated protein kinase $\mathrm{A}$ is required for differentiationdependent transcription of the decidual prolactin gene in human endometrial stromal cells. Endocrinology 138 929-937.

Thompson MA, Ginty DD, Bonni A \& Greenberg ME 1995 L-type voltage-sensitive $\mathrm{Ca}^{2+}$ channel activation regulates c-fos transcription at multiple levels. Fournal of Biological Chemistry 270 4224-4235.

Trachman JD, Huang J \& Reem GH 1995 Activation of human thymocytes induces the phosphorylation of protein tyrosine phosphatase. Comptes rendus de l'Academie Française, Paris. Sciences de la Vie/Life Sciences 318 $367-374$.

REVISED MANUSCRIPT RECEIVED 9 December 1998 